\title{
Reply to letter: "Dexamethasone for chronic subdural haematoma: a systematic review and meta-analysis"
}

\author{
Zhong Yao ${ }^{1} \cdot$ Chao You $^{1}$ (D)
}

Received: 21 September 2017 / Accepted: 29 September 2017 / Published online: 3 October 2017

(C) Springer-Verlag GmbH Austria 2017

We thank Dr. Turgut and Akhaddar for their interest and comments. They came up with several valuable points that prompted us to further elucidate the medical treatment of chronic subdural haematoma (cSDH).

As they mentioned, one study by Chan et al. [1] was not included in our systematic review. Chan et al. focused on the effect of dexamethasone on the reoperation rate rather than the recurrence. It was reported that $\mathrm{cSDH}$ patients with recurrent haematoma would be effectively treated with dexamethasone treatment once again avoiding reoperation [2]. So the reoperation rate and recurrence rate were not in full accord. In this point, we tried to distinguish the reoperation and recurrence but found it difficult because of few studies referring to their difference. Moreover, after adding Chan's study to the metaanalysis, the overall effect (RR, 0.52; 95\% CI, 0.34-0.80; $p=0.003)$ was statistically consistent with our result (RR, 0.54 ; 95\% CI, $0.33-0.88 ; p=0.01$ ).

The change of $\mathrm{cSDH}$ is a dynamic process influenced by multiple factors. We agree with that coagulation dysfunction plays an important role in the extension of cSDH. Although the osmotic and oncotic theories are questioned by some researchers, further study is needed before totally denying their effects on the cSDH. In addition, as mentioned by Turgut, bridging veins passing through the dural border cell layer may be the potential source of rebleeding.

The spontaneous regression and resolution of $\mathrm{cSDH}$ are rarely encountered. Our result also showed treatment with dexamethasone alone did not result in a better outcome. However, adjuvant dexamethasone exhibited an advantage to facilitate surgical treatment and reduce recurrence. In future studies, the adjuvant effect of dexamethasone should be the focus.

\section{References}

1. Chan DYC, Sun TFD, Poon WS (2015) Steroid for chronic subdural hematoma? A prospective phase IIB pilot randomized controlled trial on the use of dexamethasone with surgical drainage for the reduction of recurrence with reoperation. Chinese Neurosurgical Journal 1:2

2. Delgado-Lopez PD, Martin-Velasco V, Castilla-Diez JM, RodriguezSalazar A, Galacho-Harriero AM, Fernandez-Arconada O (2009) Dexamethasone treatment in chronic subdural haematoma. Neurocirugia (Astur) 20:346-359
Chao You

youchaohx@aliyun.com

1 Department of Neurosurgery, West China Hospital, Sichuan University and West China Brain Research Centre, West China Hospital, Sichuan University, No. 37 Guo Xue Xiang, Chengdu 610041, China 